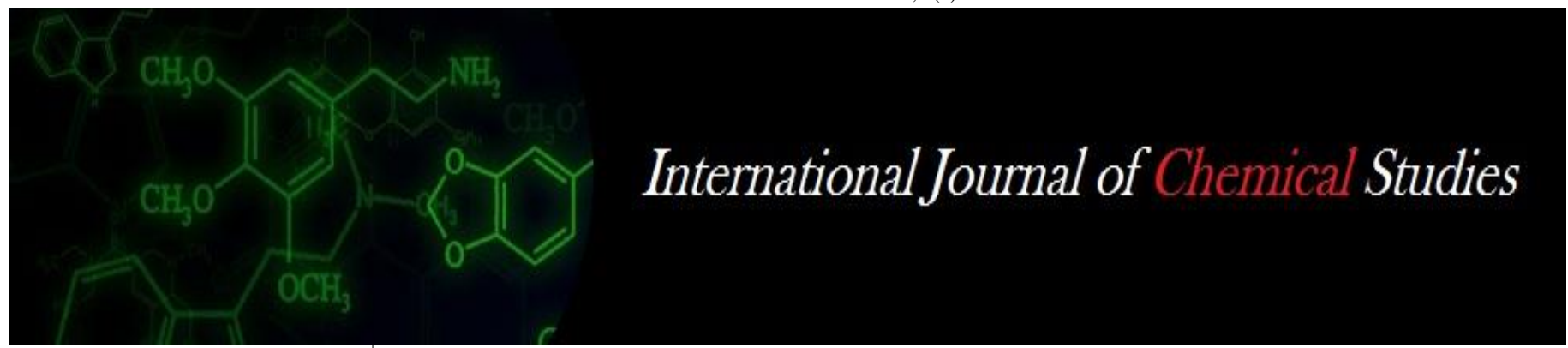

P-ISSN: 2349-8528

E-ISSN: 2321-4902

www.chemijournal.com

IJCS 2020; 8(4): 2656-2664

(C) 2020 IJCS

Received: 25-05-2020

Accepted: 27-06-2020

Mohan Kumar AB

University of Agricultural

Sciences, GKVK, Bangalore,

Karnataka, India

Vasundhara $M$

University of Agricultural

Sciences, GKVK, Bangalore,

Karnataka, India

Shyamalamma S

University of Agricultural

Sciences, GKVK, Bangalore,

Karnataka, India

Doreswamy C

University of Agricultural

Sciences, GKVK, Bangalore,

Karnataka, India

Veena S Anil

University of Agricultural

Sciences, GKVK, Bangalore,

Karnataka, India

Corresponding Author:

Mohan Kumar AB

University of Agricultural

Sciences, GKVK, Bangalore,

Karnataka, India

\section{DUS descriptor characterization of black turmeric (Curcuma caesia Roxb.) genotypes}

\author{
Mohan Kumar AB, Vasundhara M, Shyamalamma S, Doreswamy C and \\ Veena S Anil
}

DOI: $\underline{\text { htps://doi.org/10.22271/chemi.2020.v8.i4ae.10042 }}$

\begin{abstract}
A study was carried out at ICAR KVK Chamarajanagar to characterize thirty three black turmeric genotypes for twenty five characters as per the multiscale scores guidelines of DUS. Among the characters studied, four characters viz., leaf margin, number of mother rhizome, coma bract colour and bract tip colour were found monomorphic, eleven were dimporphic and ten were polymorphic. Vigour of the plant viz., tall plants, long petiole \& lamina length and broader leaves were considered as a morphological characters for selection of adoptable genotypes (GKM-2, GKB-3, GKJ-5, GOK-19, GAP20, GMI-22, GNF-27, GMR-31) to local conditions. Pseudo stem anthocyanin colouration of GAB12, GMI22 and GNP31 is unique. All the genotypes had coloured coma bract and exhibited rose bract tip. Variations in the inner core colour of black turmeric rhizomes have been emphasized. Greenish blue \& pale green colour of the rhizome inner core can be use as a marker for identification of the GKJ5 \& GMV6 genotypes respectively. Genotypes GAB-13 and GAB-14 were found to be short duration type of less than 210 days; earliness can be a marker for GAB-13 and GAB-14 genotypes. The variations observed using DUS characters offers a bright scope for selection based on desirable morphological traits, which can be potential in utilization for trait specific selection.
\end{abstract}

Keywords: Black turmeric, monomorphic, dimorphic, polymorphic, pigmentation, coma bract, distinctiveness uniformity and stability (DUS)

\section{Introduction}

Curcuma caesia Roxb., is an important, lesser known, non-conventional medicinal plant belongs to Zingiberaceae family. Native to North-East and central India, distributed to Java, Myanmar and rarely found in Madhya Pradesh, Jharkhand, Chhattisgarh, Orissa and other parts of South India.

Medicinal potentiality of Curcuma caesia Roxb., is varied, rhizomes useful in treating several diseases like diabetes, high cholesterol, abdominal pains, menstrual disorder, Wounds, eczema, psoriasis, Jaundice, Inflammations, Cancerous Symptoms and as a blood purifying activity (Arulmozhi et al., 2006) [3], bronchodilating activity (Paliwal et al., 2011) [16], antioxidant activity (Mangla et al., 2010) ${ }^{[10]}$, anxiolytic and CNS depressant activity, locomotor depressant, anticonvulsant (Karmakar et al., 2011) ${ }^{[9]}$, anthelmintic activity (Gill et al., 2011) ${ }^{[7]}$, anti-bacterial activity (Rajamma et al.,2012) ${ }^{[19]}$, anti-ulcer activity (Das et al., 2012) ${ }^{[4]}$.

Black turmeric plants used for tantric sadhana and medication by tribal people. Northern tribes use rhizomes as a talisman to keep the evil spirits away, while in West Bengal it finds an important place in traditional system of medicine and is also used as a substitute for turmeric in fresh state (Wikipedia, 2017) ${ }^{[22]}$.

Presently National Medicinal Plant Board (NMPB) of India has listed this plant as crucially vulnerable species. Ministry of Environment imposes restrictions on export without permission of the legal competent authorities. Curcuma caesia Roxb. has been categorized as endangered due to great demand, indiscriminate exploitation and limited cultivation (Neha et al., 2014) ${ }^{[14]}$. Existence of wide variability among the turmeric genotypes with respect to yield attributes and quality characters has been reported by many researchers (Anandaraj et al., 2014; Prasath et $a l ., 2016)^{[2,18]}$. Genetic variation has implications for the conservation at the species level. 
Systematic and detailed characterization of genotypes is required for better conservation. Morphological characterization is an important tool even in the era of molecular characterization because of its reliability and easy identification with less resources for certain stable characters unaltered with environmental interactions. The guidelines of Protection of Plant Varieties and Farmers Right Act (2009) of India help in categorizing the morphological characters which are measured quantitatively and qualitatively. Therefore, the present study was undertaken to characterize a set of 33 Black turmeric genotypes collected from provenance of the country, for different morphological and rhizome characters based on DUS guidelines as a measure of conservation of the species.

\section{Materials and Methods}

Thirty three Black turmeric genotypes were collected from the provenance of the country (Table 1). The genotypes were characterized at ICAR-KVK Chamarajanagar, Karnataka. Crop was grown during two consecutive seasons of 2018-19 and 2019-20 in a randomized block design with three replications. 54 rhizomes were planted in each plot $(3 \mathrm{~m} \mathrm{X}$ $2 \mathrm{~m}$ ) at spacing of $30 \mathrm{~cm} X 30 \mathrm{~cm}$. The field was maintained under uniform recommended cultural practices (Jayashree et al., 2015) ${ }^{[8]}$. Five plants of uniform size and vigour was selected for recording observations. Genotypes were evaluated for 25 DUS traits viz., Plant: pseudostem habit, plant height $(\mathrm{cm})$, number of shoots, number of leaves on main stem, Plant: leaf disposition, petiole length $(\mathrm{cm})$, leaf lamina length $(\mathrm{cm})$, leaf lamina width $(\mathrm{cm})$, dorsal leaf colour, ventral leaf colour, leaf mid-rib colour, leaf venation pattern, leaf margin, pseudostem anthocyanin colouration, coma bract colour, bract tip colour, rhizome habit, rhizome shape, rhizome internode pattern, status of tertiary rhizome, primary rhizome length, number of mother rhizome, primary rhizome inner core colour, duration and dry recovery (\%). The assessment of characters was done at $150^{\text {th }}$ days after planting for vegetative characters and after harvest for rhizome characters (PPV \& FRA, 2009) ${ }^{[17]}$. Observations based on colour were recorded under natural light using Royal Horticultural Society (RHS) Colour chart.

\section{Results and Discussion}

Among the 33 genotypes studied, considerable variation was recorded for all the important characters. Out of 25 characters assessed based on DUS descriptors, four characters viz., leaf margin, number of mother rhizome, coma bract colour and bract tip colour were found to be monomorphic, eleven were dimporphic and ten were polymorphic characteristics. Classically Black turmeric genotypes are grouped based on pseudo stem height, duration and dry recovery. Variability among the genotypes to certain morphological characters like Plant: leaf disposition, leaf venation pattern, pseudostem anthocyanin colouration and leaf midrib colour also be considered for grouping the genotypes. Grouping can also be done for rhizome habit, internode pattern and rhizome colour as wide variation on primary rhizome inner core colour is also documented among the selected 33 genotypes (Table 2).

\section{Plant and leaf characteristics}

Among the genotypes studied, the Pseudostem habit in twelve genotypes was compact and twenty one were open. Plant height being a polymorphic characteristic among the genotypes twenty four under short statures $(<85 \mathrm{~cm})$, four under medium $(80$ to $100 \mathrm{~cm})$ and five were under tall category $(100.30$ to $103.61 \mathrm{~cm})$. Number of shoots and leaves on main stem was recorded as dimorphic characters, number of shoots was medium in GKB-4, other 32 genotypes had shoots of more than five. More number of leaves on main stem was recorded in thirty genotypes with range of 5-10 leaves, three genotypes had few leaves $<5$ in the main stem. Leaf disposition is unique in 04 genotypes viz., GNK25, GNU26, GAD32 \& GJG35 horizontal $\left(>85^{\circ}\right)$. Twenty genotypes exhibits semi-errect $\left(45^{0}-85^{0}\right)$ and nine erect $\left(<45^{\circ}\right)$ type leaf disposition. Most of the high yielding genotypes found in erect type of leaf disposition group, which mainly contributed to larger light capture per unit leaf area due to a reduced aggregation of leaf area around the stem. Polymorphic leaf characters like leaf petiole length, lamina length and leaf width has a direct influence on overall performance of the plant. Short, intermediate and long leaf petiole length was observed in equal number (eleven each) of studied genotypes. Leaf lamina length is long $(>40 \mathrm{~cm})$ in nine, medium $(30-40 \mathrm{~cm})$ in thirteen and short $(<30 \mathrm{~cm})$ in eleven genotypes. Leaf width was narrow $(<10 \mathrm{~cm})$ in nine, medium $(10-15 \mathrm{~cm})$ in thirteen, while remaining eleven were broad $(>15 \mathrm{~cm})$. It was observed that genotypes GKM-2, GKB-3, GKJ-5, GMI-22, GOK-19, GAP-20, GNF-27, GMR31 has shown better vegetative growth viz., taller plants, longer petiole \& lamina length and wider leaf width, indicating the adoptability of these genotypes to the local conditions. Vigourness of the plant can be considered as a morphological character for slelection of adoptable genotypes to local conditions. Leaf venation pattern was close in twenty seven genotypes, broad in six genotypes viz.,.GKM-1, GKM2, GKB-3, GKJ-5, GMF-21, GMI-22. Distant leaf venation pattern characteristic can be used as markers for easy differentiation of genotypes for good yield attributes. Because all the six genotypes which were exhibited broad leaf venation pattern has shown vigour in growth and comparatively good yield attributes compared to other genotypes. This might be due to broader leaf venation supports exposure of maximum leaf surface area to the sunlight, in turn which helps to accumulate more photosynthates. Leaf margin was unique all the genotypes showing even leaf margin pattern.

Pseudostem anthocyanin colouration, leaf colour on ventral \& dorsal side was observed to be dimorphic as different shade was observed in genotypes. Different shades of purple red colour of pseudostem anthocyanin was observed in ten genotypes and absent in twenty four genotypes, these genotypes exhibits yellow green psuedostem colouration (Table 4B). Leaf colour on the ventral side (upper) ranges from Green to dark green, eight genotypes, leaves are dark green colour and remaining twenty five genotypes, the leaves were found normal green colour. Dorsal side (lower) leaf colour ranges from light green to dark green, sixteen genotypes, the leaves lower side are light green in colour and remaining seventeen genotypes were found normal green in colour. Leaf midrib colour is the unique identification character in Curcuma caesia Roxb. species. Varied shades of Violet blue (N92A), Purple (N77A, 79A, N79A, N79B) and Greyed purple (N187A) leaf midrib colour was observed (Table 4A). Three genotypes exhibits greyed purple (dark purplish green) leaf-midrib colour viz., GMI-22, GKK-30, GMR-31, this can be considered as an identification marker for these genotype. Purple group (N77A, 79A, N79A, N79B) was observed in nineteen genotypes and Violet blue group (N92A) colour in eleven genotypes. Similar study on turmeric characterization based on DUS character was reported by Deb and Chakrobarty, (2017) ${ }^{[5]}$ and Aarthi et al. (2018) ${ }^{[1]}$. 


\section{Floral characteristics}

All the genotypes had flowers with coloured coma bract and exhibited rose bract tip colour and these genotypes were found monomorphic in nature. These important charecteristics will be a unique morphology for identification of the species C. caesia Roxb. The above observations are supported by the findings of Sharma et al., 2011 ${ }^{[20]}$ and Aarthi et al., $2018^{[1]}$. The variability in coma bract colour is evident across the Curcuma spp. and inter species variability in coma bract is evident in case of $C$. longa.

\section{Rhizome characteristics}

Rhizome being the economical part in Black turmeric, the variation range is more among the genotypes studied. Rhizome habit is characterized based on the primary attachment to the mother rhizome. Fifteen genotypes characterized under loose habit, twelve were compact and six were intermediate. Among the genotypes studied six were having straight rhizomes and twenty seven were curved. Price is fixed based on the length of primary rhizome; wide variability is seen in rhizome length. Twenty nine were recorded medium length $(5$ to $10 \mathrm{~cm}$ ) and four were found long with more than $10 \mathrm{~cm}$ length and none were categorized under short category. Mother rhizome is used as a planting material in turmeric to get good yield (Padmadevi et al., 2012) [15] and has high secondary metabolites because of degradation of starch, so mother rhizome influence the chemical constituent and genotypes differs with number of mother rhizome per plant (Neeraja et al., 2017) ${ }^{[13]}$. The variation was observed with respect to the size of the mother rhizomes. All thirty three genotypes were having more than three mother rhizomes per plant. Rhizome internode pattern was close in eighteen genotypes and fifteen had distant internode venation pattern. Presence of tertiary rhizomes have positive effect on rhizome yield at genotypic level (Singh et al., 2012) ${ }^{[21]}$. Tertiary rhizomes were absent in ten genotypes and present in twenty three genotypes.

The inner core colour of the rhizomes decides the quality and chemical constituent of the rhizome. Wide variation was noticed in the inner core colour of black turmeric rhizomes. The colour was categorized based on RHS colour chart. Different shades of blue and green colour were noticed. Rhizome inner core colour majorly falls into Blue green group and Green group (Table 4C). Twelve genotypes were categorized as light blue colour, sixteen were found under strong blue colour, three were moderate blue colour and one genotype each under greenish blue \& pale green colour viz.,GKJ-5 \& GMV-6 respectively. Greenish blue \& pale green colour of the rhizome inner core can be used as a marker for identification of the respective genotypes. Variation in rhizome core colour of turmeric was reported by Mishra et al., 2015; Aarthi et al., $2018^{[1]}$.

Earliness is a desirable character; early varieties are suitable for areas with serious irrigation problems. Genotypes GAB-13 and GAB-14 are short duration type (less than 210 Days), this morphological characteristic can be a marker for respective genotypes. Six genotypes are medium (211-240 days) and twenty five are long duration types (>241 days). Percentage of dry recovery was low in five genotypes, intermediate in eighteen genotypes and in another ten genotypes it was high recovery with more than 20 percent. Similar observation was made in ginger using DUS guidelines to characterize the soma clones (Dev, 2013) ${ }^{[6]}$ and in fifteen turmeric genotypes by Aarthi et al. (2018) ${ }^{[1]}$.

Apart from DUS characters, there were some noticeable variation in the selected genotypes which can act as morphological markers for identification of genotypes; they are collar girth of the pseudo stem, colour intensity of leaf midrib, intensity rhizome inner core colour and lodging of the plant at 220 DAS.

Collar girth of the pseudo stem has significant variation, among the genotypes GKM-2, GKB-3, GKJ-5, GMI-22, GMR-31 has higher pseudo stem collar girth. Thick colour intensity of leaf midrib is noticed in genotype GMR-31. Rhizome inner core colour intensity ranges from light blue to strong blue. In between various shades of very light blue (GAB13), very pale blue (GKM2), moderate blue (GKM1), very light greenish blue (GKJ5), very pale purplish blue (GAK14) and very pale green (GMV6) occurs. Lodging of the plant at final stage of the crop (after 220DAS) was observed in five genotypes viz., GKM-2, GKB-3, GKJ-5, GMI-22, GMR-31, was absent in remaining twenty eight genotypes. Lodged genotypes were excellent in vegetative growth; which indicates the acclimatization of genotypes to the local condition. Majority of the lodged genotypes were comparatively high yielder than the non lodged genotypes.

Table 1: Details of black turmeric genotypes collected from provenance of the country

\begin{tabular}{|c|c|c|c|c|c|c|}
\hline$\#$ & Genotype & Place of origin & Latitude & Longitude & Altitude (m) & State \\
\hline 01 & GKM-1 & Mijar, & $13^{\circ} 4^{\prime} 7.6764^{\prime \prime} \mathrm{N}$ & $74^{\circ} 59^{\prime} 36.9564^{\prime \prime} \mathrm{E}$ & 147 & Karnataka \\
\hline 02 & GKM-2 & Mangalore & $12^{\circ} 55^{\prime} 2.03^{\prime \prime} \mathrm{N}$ & $74^{\circ} 51^{\prime} 21.71^{\prime \prime E}$ & 22 & Karnataka \\
\hline 03 & GKB-3 & Bangalore & $12.9716^{\circ} \mathrm{N}$ & $77.5946^{\circ} \mathrm{E}$ & 920 & Karnataka \\
\hline 04 & GKB-4 & Sanjeevini vatika & $13.0801^{\circ} \mathrm{N}$ & $77.5785^{\circ} \mathrm{E}$ & 924 & Karnataka \\
\hline 05 & GKJ-5 & Joida & $15.1688^{\circ} \mathrm{N}$ & $74.4848^{\circ} \mathrm{E}$ & 532 & Karnataka \\
\hline 06 & GMV-6 & Vidarbha-Gadehirolli & $21.1286^{\circ} \mathrm{N}$ & $79.0964^{\circ} \mathrm{E}$ & 1000 & Maharashtra \\
\hline 07 & GBS-8 & Samastipur & $25.8629679 \mathrm{~N}$ & $85.7810263 \mathrm{E}$ & 53 & Bihar \\
\hline 08 & GBH-9 & Hajipur & $25.6858392 \mathrm{~N}$ & $85.2145907 \mathrm{E}$ & 56 & Bihar \\
\hline 09 & GGR-10 & Rajkote & $22^{\circ} 17^{\prime} 30 \mathrm{~N}$ & $70^{\circ} 47^{\prime} 36 \mathrm{E}$ & 252 & Gujarat \\
\hline 10 & GAB-11 & Bhoka Ghat Forest & $26.2006^{\circ} \mathrm{N}$ & $92.9376^{\circ} \mathrm{E}$ & 76 & Assam \\
\hline 11 & GAB-12 & Bijuli & $28.0312^{\circ} \mathrm{N}$ & $82.9555^{\circ} \mathrm{E}$ & 97 & Assam \\
\hline 12 & GAB-13 & Bokoliya & $26.0564^{\circ} \mathrm{N}$ & $93.1955^{\circ} \mathrm{E}$ & 600 & Assam \\
\hline 13 & GAK-14 & Killing Basti & $26.8140^{\circ} \mathrm{N}$ & $82.7630^{\circ} \mathrm{E}$ & 680 & Assam \\
\hline 14 & GMW-15 & Wakhro & $23^{\circ} 43^{\prime} 2.6256^{\prime \prime} \mathrm{N}$ & $92^{\circ} 43^{\prime} 5.2212^{\prime \prime} \mathrm{E}$ & 1619 & Mizoram \\
\hline 15 & GMK16 & Kolasib & $24.2246^{\circ} \mathrm{N}$ & $92.6760^{\circ} \mathrm{E}$ & 722 & Mizoram \\
\hline 16 & GMA-17 & Aizwal & $23.727106^{\circ} \mathrm{N}$ & $92.717636^{\circ} \mathrm{E}$ & 1132 & Mizoram \\
\hline 17 & GOK-18 & Khurda & $20.1301^{\circ} \mathrm{N}$ & $85.4788^{\circ} \mathrm{E}$ & 75 & Odisha \\
\hline 18 & GOK-19 & Koraput & $18.82^{\circ} \mathrm{N}$ & $82.72^{\circ} \mathrm{E}$ & 870 & Odisha \\
\hline 19 & GAP-20 & Pasighat Area & $28.0619^{\circ} \mathrm{N}$ & $95.3260^{\circ} \mathrm{E}$ & 153 & Arunachal Pradesh \\
\hline 20 & GMI-21 & Manipur - Forest & $24^{\circ} 48^{\prime} 50.2812^{\prime \prime} \mathrm{N}$ & $93^{\circ} 57^{\prime} 1.0044^{\prime \prime} \mathrm{E}$ & 900 & Manipur \\
\hline
\end{tabular}




\begin{tabular}{|l|c|c|c|c|c|c|}
\hline 21 & GMT-22 & Imphal & $24.8170^{\circ} \mathrm{N}$ & $93.9368^{\circ} \mathrm{E}$ & 786 & Manipur \\
\hline 22 & GMS-24 & Sagar & $23.8388^{\circ} \mathrm{N}$ & $78.7378^{\circ} \mathrm{E}$ & 427 & Madhya Pradesh \\
\hline 23 & GNK-25 & Kohima & $25.6751^{\circ} \mathrm{N}$ & $94.1086^{\circ} \mathrm{E}$ & 1444 & Nagaland \\
\hline 24 & GNU-26 & Uhkagoronga Hill & $25^{\circ} 54^{\prime} 22.5612^{\prime \prime} \mathrm{N}$ & $93^{\circ} 43^{\prime} 39.3312^{\prime \prime} \mathrm{E}$ & 3827 & Nagaland \\
\hline 25 & GNF-27 & Nepal - Forest & $27^{\circ} 42^{\prime} 2.7684^{\prime \prime} \mathrm{N}$ & $85^{\circ} 18^{\prime} 0.5040^{\prime \prime} \mathrm{E}$ & 330 & Nepal \\
\hline 26 & GKT-29 & Thrissur-Vellanikara & $10.5452^{\circ} \mathrm{N}$ & $76.2740^{\circ} \mathrm{E}$ & 22 & Kerala \\
\hline 27 & GKK-30 & IISR Kozhikode & $11.2588^{\circ} \mathrm{N}$ & $75.7804^{\circ} \mathrm{E}$ & 1 & Kerala \\
\hline 28 & GMR-31 & Ri-Bhoi & $25.8432^{\circ} \mathrm{N}$ & $91.9856^{\circ} \mathrm{E}$ & 485 & Meghalaya \\
\hline 29 & GAD-32 & Dolamora Borpung & $26^{\circ} 14^{\prime} 38.9616^{\prime \prime} \mathrm{N}$ & $92^{\circ} 32^{\prime} 16.2312^{\prime \prime} \mathrm{E}$ & 615 & Assam \\
\hline 30 & GNP-33 & Peren & $25.5125^{\circ} \mathrm{N}$ & $93.7391^{\circ} \mathrm{E}$ & 1445 & Nagaland \\
\hline 31 & GBC-34 & Champaran & $27.1543^{\circ} \mathrm{N}$ & $84.3542^{\circ} \mathrm{E}$ & 62 & Bihar \\
\hline 32 & GJG-35 & Godda & $24.8255^{\circ} \mathrm{N}$ & $87.2135^{\circ} \mathrm{E}$ & 87 & Jharkhand \\
\hline 33 & GNP-36 & Phek & $25.6634^{\circ} \mathrm{N}$ & $94.4703^{\circ} \mathrm{E}$ & 1524 & Nagaland \\
\hline
\end{tabular}

Table 2: Grouping traits of 33 black turmeric genotypes based on DUS descriptors

\begin{tabular}{|c|c|c|c|c|c|c|}
\hline $\begin{array}{l}\text { SI } \\
\text { no }\end{array}$ & Characteristic & $\begin{array}{c}\text { Type of } \\
\text { Assessment }\end{array}$ & State & Score & $\begin{array}{c}\text { No. of } \\
\text { genotypes }\end{array}$ & Reference genotypes \\
\hline \multirow[b]{2}{*}{1} & \multirow[b]{2}{*}{$\begin{array}{l}\text { Plant: Pseudo stem } \\
\text { habit }\end{array}$} & \multirow[b]{2}{*}{ VG } & Compact & 1 & 12 & $\begin{array}{l}\text { GKM-1, GKM-2, GKB-3, GKJ-5, GBS-8, GAB-13, GAB-14, GMW- } \\
\text { 15, GMI-22, GMS-24, GMR-31, GNP-33. }\end{array}$ \\
\hline & & & Open & 9 & 21 & $\begin{array}{l}\text { GKB-4, GMV-6, GBH-9, GGR-10, GAB-11, GAB-12, GMK-16, } \\
\text { GMA-17, GOK-18, GOK-19, GAP-20, GMF-21, GNK-25, GNU-26, } \\
\text { GNF-27, GKT-29, GKK-30, GAD-32, GBC-34, GJG-35, GNP-36. }\end{array}$ \\
\hline \multirow[t]{3}{*}{2} & \multirow[t]{3}{*}{ Plant height $(\mathrm{cm})$} & \multirow[t]{3}{*}{ MS } & Short $(<85)$ & 3 & 24 & $\begin{array}{l}\text { GKM-1, GKB-4, GMV-6, GBS-8, GBH-9, GGR-10,GAB-13, GAB- } \\
\text { 14,GMW-15, GMK-16, GMA-17, GOK-18, GMF-21, GMS-24, GNK- } \\
\text { 25, GNU-26, GNF-27, GKT-29, GKK-30, GAD-32, GNP-33, GBC-34, } \\
\text { GJG-35, GNP-36 }\end{array}$ \\
\hline & & & $\begin{array}{l}\text { Medium }(85- \\
100)\end{array}$ & 5 & 04 & GAB-11, GAB-12, GOK-19, GAP-20. \\
\hline & & & Tall $(>100)$ & 7 & 05 & GKM-2, GKB-3, GKJ-5, GMI-22, GMR-31. \\
\hline \multirow[b]{3}{*}{3} & \multirow[b]{3}{*}{ Plant: No. of shoots } & \multirow[b]{3}{*}{ MG } & Few $(<3)$ & 1 & 00 & - \\
\hline & & & Medium (3-5) & 3 & 01 & GKB-4 \\
\hline & & & Many $(>5)$ & 5 & 32 & $\begin{array}{l}\text { GKM-1, GKM-2, GKB-3, GKJ-5, GMV-6, GBS-8, GBH-9, GGR-10, } \\
\text { GAB-11, GAB-12, GAB-13, GAB-14,GMW-15, GMK-16, GMA-17, } \\
\text { GOK-18, GOK-19, GAP-20, GMF-21,GMI-22, GMS-24, GNK-25, } \\
\text { GNU-26, GNF-27, GKT-29, GKK-30, GMR-31, GAD-32, GNP-33. } \\
\text { GBC-34, GJG-35, GNP-36. }\end{array}$ \\
\hline \multirow{3}{*}{4} & \multirow{3}{*}{$\begin{array}{l}\text { Plant: Number of } \\
\text { leaves on main } \\
\text { shoot }\end{array}$} & \multirow{3}{*}{ MG } & Few $(<5)$ & 3 & 03 & GKB-4, GAB-11, GOK-18. \\
\hline & & & $\begin{array}{c}\text { Intermediate }(5- \\
10)\end{array}$ & 5 & 30 & $\begin{array}{l}\text { GKM-1, GKM-2, GKB-3, GKJ-5, GMV-6, GBS-8, GBH-9, GGR-10, } \\
\text { GAB-12, GAB-13, GAB-14, GMW-15, GMK-16, GMA-17, GOK-19, } \\
\text { GAP-20, GMF-21, GMI-22, GMS-24, GNK-25, GNU-26, GNF-27, } \\
\text { GKT-29, GKK-30, GMR-31, GAD-32, GNP-33. GBC-34, GJG-35, } \\
\text { GNP-36. }\end{array}$ \\
\hline & & & Many $(>10)$ & 7 & 00 & - \\
\hline $\begin{array}{l}\text { Sl } \\
\text { no }\end{array}$ & Charecteristic & $\begin{array}{c}\text { Type of } \\
\text { Assessment }\end{array}$ & State & Score & $\begin{array}{c}\text { No. of } \\
\text { genotypes }\end{array}$ & Reference genotypes \\
\hline \multirow{3}{*}{5} & \multirow{3}{*}{$\begin{array}{l}\text { Plant: Leaf } \\
\text { disposition }\end{array}$} & \multirow{3}{*}{ VG } & Erect $\left(<45^{\circ}\right)$ & 3 & 09 & $\begin{array}{l}\text { GKM-1, GKM-2, GKB-3, GKJ-5, GBS-8, GBH-9, GGR-10, GAB-12. } \\
\text { GMI-22. }\end{array}$ \\
\hline & & & $\begin{array}{c}\text { Semi-erect }\left(45^{\circ}-\right. \\
\left.85^{\circ}\right)\end{array}$ & 5 & 20 & $\begin{array}{l}\text { GKB-4, GMV-6, GAB-11, GAB-13, GAB-14, GMW-15, GMK-16, } \\
\text { GMA-17, GOK-18, GOK-19, GAP-20, GMF-21, GMS-24, GNF-27, } \\
\text { GKT-29, GKK-30, GMR-31, GNP-33. GBC-34, GNP-36. }\end{array}$ \\
\hline & & & $\begin{array}{c}\text { Horizontal } \\
\left(>85^{\circ}\right)\end{array}$ & 7 & 04 & GNK25, GNU26, GAD32, GJG35 \\
\hline \multirow{3}{*}{6} & \multirow{3}{*}{$\begin{array}{c}\text { Leaf: Petiole length } \\
(\mathrm{cm})\end{array}$} & \multirow{3}{*}{ MS } & Short $(<15)$ & 3 & 11 & $\begin{array}{l}\text { GKB-4, GBS-8, GBH-9, GMW-15, GMK-16, GAP-20, GMF-21, } \\
\text { GNU-26, GKK-30, GNP-33, GBC-34. }\end{array}$ \\
\hline & & & $\begin{array}{l}\text { Intermediate } \\
(15-25)\end{array}$ & 5 & 11 & $\begin{array}{l}\text { GKM-1, GMV-6, GAB-11, GAB-12, GAB-13, GOK-18, GNK-25, } \\
\text { GKT-29, GAD-32, GJG-35, GNP-36. }\end{array}$ \\
\hline & & & Long $(>25)$ & 7 & 11 & $\begin{array}{l}\text { GKM-2, GKB-3, GKJ-5, GGR-10, GAB-14, GMA-17, GOK-19, GMI- } \\
\text { 22, GMS-24, GNF-27, GMR-31. }\end{array}$ \\
\hline \multirow{3}{*}{7} & \multirow{3}{*}{$\begin{array}{l}\text { Leaf: Lamina } \\
\text { length }(\mathrm{cm})\end{array}$} & \multirow{3}{*}{ MS } & Short $(<30)$ & 3 & 11 & $\begin{array}{l}\text { GKB-4, GBS-8, GBH-9, GMW-15, GMK-16, GAP-20, GMF-21, } \\
\text { GNU-26, GKK-30, GNP-33, GBC-34. }\end{array}$ \\
\hline & & & Medium (30-40) & 5 & 13 & $\begin{array}{l}\text { GKM-1, GMV-6, GAB-11, GAB-12, GAB-13, GAB-14, GMA-17, } \\
\text { GOK-18, GNK-25, GKT-29, GAD-32, GJG-35, GNP-36. }\end{array}$ \\
\hline & & & Long $(>40)$ & 7 & 09 & $\begin{array}{l}\text { GKM-2, GKB-3, GKJ-5, GGR-10, GOK-19, GMI-22, GMS-24, GNF- } \\
\text { 27, GMR-31. }\end{array}$ \\
\hline \multirow{3}{*}{8} & \multirow{3}{*}{$\begin{array}{l}\text { Leaf: Lamina width } \\
(\mathrm{cm})\end{array}$} & \multirow{3}{*}{ MS } & Narrow $(<10)$ & 3 & 09 & $\begin{array}{l}\text { GKB-4, GBS-8, GBH-9, GMK-16, GAP-20, GNU-26, GKK-30, GNP- } \\
\text { 33, GBC-34. }\end{array}$ \\
\hline & & & Medium (10-15) & 5 & 13 & $\begin{array}{l}\text { GKM-1, GMV-6, GAB-11, GAB-12, GAB-13, GMW-15, GOK-18, } \\
\text { GMF-21, GNK-25, GKT-29, GAD-32, GJG-35, GNP-36. }\end{array}$ \\
\hline & & & Broad $(>15)$ & 7 & 11 & $\begin{array}{l}\text { GKM-2, GKB-3, GKJ-5, GGR-10, GAB-14, GMA-17, GOK-19, GMI- } \\
\text { 22, GMS-24, GNF-27, GMR-31. }\end{array}$ \\
\hline
\end{tabular}




\begin{tabular}{|c|c|c|c|c|c|c|}
\hline \begin{tabular}{l|} 
SI \\
no
\end{tabular} & Characteristic & \begin{tabular}{|c|} 
Type of \\
Assessment
\end{tabular} & States & Score & $\begin{array}{c}\text { No. of } \\
\text { genotypes }\end{array}$ & Reference genotypes \\
\hline & \multirow{3}{*}{$\begin{array}{c}\text { Leaf: colour on } \\
\text { dorsal side (Lower) }\end{array}$} & \multirow{3}{*}{ VG } & Light green & 3 & 16 & $\begin{array}{l}\text { GKM-2, GKB-3, GKB-4, GKJ-5, GMV-6, GBH-9, GGR-10, GAB-12, } \\
\text { GMK-16, GMA-17, GOK-18, GOK-19, GAP-20, GMF-21, GMI-22, } \\
\text { GMS-24. }\end{array}$ \\
\hline 9 & & & Green & 5 & 17 & $\begin{array}{l}\text { GKM-1, GBS-8, GAB-11, GAB-13, GAB-14, GMW-15, GNK-25, GNU- } \\
\text { 26, GNF-27, GKT-29, GKK-30, GMR-31, GAD-32, GNP-33. GBC-34, } \\
\text { GJG-35, GNP-36. }\end{array}$ \\
\hline & & & Dark green & 7 & 00 & 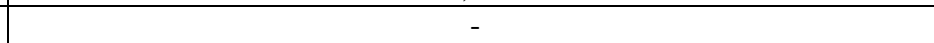 \\
\hline \multirow[t]{2}{*}{10} & \multirow[t]{2}{*}{$\begin{array}{l}\text { Leaf: colour on } \\
\text { ventral side } \\
\text { (Upper) }\end{array}$} & \multirow[t]{2}{*}{ VG } & Green & 5 & 25 & $\begin{array}{c}\text { GKM-1, GKM-2, GKB-4, GMV-6, GBH-9, GGR-10, GAB-11, GAB-12, } \\
\text { GAB-14, GMK-16, GMA-17, GOK-18, GOK-19, GAP-20, GMF-21, } \\
\text { GMI-22, GNK-25, GNF-27, GKT-29, GKK-30, GMR-31, GAD-32, } \\
\text { GNP-33. GBC-34, GNP-36. }\end{array}$ \\
\hline & & & Dark green & 7 & 08 & GKB-3, GKJ-5, GBS-8, GAB-13, GMW-15, GMS-24, GNU-26, GJG-35 \\
\hline \multirow[t]{2}{*}{11} & \multirow[t]{2}{*}{ Leaf: Margin } & \multirow[t]{2}{*}{ VG } & Even & 3 & 33 & $\begin{array}{l}\text { GKM-1, GKM-2, GKB-3, GKB-4, GKJ-5, GMV-6, GBS-8, GBH-9, } \\
\text { GGR-10, GAB-11, GAB-12,GAB-13, GAB-14,GMW-15, GMK-16, } \\
\text { GMA-17, GOK-18, GOK-19, GAP-20, GMF-21,GMI-22,GMS-24, GNK } \\
\text { 25, GNU-26, GNF-27, GKT-29, GKK-30, GMR-31, GAD-32, GNP-33. } \\
\text { GBC-34, GJG-35, GNP-36. }\end{array}$ \\
\hline & & & Wavy & 5 & 00 & - \\
\hline \multirow[t]{2}{*}{12} & \multirow[t]{2}{*}{$\begin{array}{l}\text { Leaf: Venation } \\
\text { pattern }\end{array}$} & \multirow[t]{2}{*}{ MS } & Close & 3 & 27 & $\begin{array}{l}\text { GKB-4, GMV-6, GBS-8, GBH-9, GGR-10, GAB-11, GAB-12, GAB-13, } \\
\text { GAB-14, GMW-15, GMK-16, GMA-17, GOK-18, GOK-19, GAP-20, } \\
\text { GMS-24, GNK-25, GNU-26, GNF-27, GKT-29, GKK-30, GMR-31, } \\
\text { GAD-32, GNP-33. GBC-34, GJG-35, GNP-36. }\end{array}$ \\
\hline & & & Distant & 5 & 06 & GKM-1, GKM-2, GKB-3, GKJ-5, GMF-21, GMI-22. \\
\hline \multirow[t]{2}{*}{13} & \multirow{2}{*}{$\begin{array}{l}\text { Pseudo stem: } \\
\text { Anthocyanin } \\
\text { colouration }\end{array}$} & \multirow[t]{2}{*}{ VG } & Absent & 1 & 23 & $\begin{array}{l}\text { GKM-1, GKM-2, GKJ-5, GMV-6, GGR-10, GAB-11, GMW-15, GMA- } \\
\text { 17, GOK-18, GOK-19, GAP-20, GMF-21, GMS-24, GNK-25, GNU-26, } \\
\text { GNF-27, GKT-29, GKK-30, GAD-32, GNP-33. GBC-34, GJG-35, GNP- } \\
36 .\end{array}$ \\
\hline & & & Present & 9 & 10 & $\begin{array}{l}\text { GKB-3, GKB-4, GBS-8, GBH-9, GAB-12, GAB-13, GAB-14, GMK-16, } \\
\text { GMI-22, GMR-31. }\end{array}$ \\
\hline \begin{tabular}{l|}
$\mathrm{SI}$ \\
no
\end{tabular} & Characteristic & \begin{tabular}{|c|} 
Type of \\
Assessment
\end{tabular} & States & Score & $\begin{array}{c}\text { No. of } \\
\text { genotypes }\end{array}$ & Reference genotypes \\
\hline \multirow{3}{*}{14} & \multirow{3}{*}{ Leaf: midrib colour } & \multirow{3}{*}{ VG } & $\begin{array}{l}\text { Violet blue } \\
\text { group }\end{array}$ & 1 & 11 & $\begin{array}{l}\text { GKM-1, GKB-4, GKJ-5, GBS-8, GMW-15, GMS-24, GNK-25, GNF-27, } \\
\text { GKT-29, GAD-32, GNP-36. }\end{array}$ \\
\hline & & & Purple group & 3 & 19 & $\begin{array}{c}\text { GKM-2, GKB-3, GMV-6, GBH-9, GGR-10, GAB-11, GAB-12, GAB-13 } \\
\text { GAB-14, GMK-16, GMA-17, GOK-18, GOK-19, GAP-20, GMF-21, } \\
\text { GNU-26, GNP-33, GBC-34, GJG-35. }\end{array}$ \\
\hline & & & \begin{tabular}{|c|} 
Greyed \\
purple group
\end{tabular} & 5 & 03 & GMI-22, GKK-30, GMR-31, \\
\hline \multirow[b]{2}{*}{15} & \multirow[b]{2}{*}{ Coma bract: colour } & \multirow[b]{2}{*}{ VS } & White & 1 & 00 & - \\
\hline & & & Coloured & 9 & 33 & $\begin{array}{l}\text { GKM-1, GKM-2, GKB-3, GKB-4, GKJ-5, GMV-6, GBS-8, GBH-9, } \\
\text { GGR-10, GAB-11, GAB-12, GAB-13, GAB-14, GMW-15, GMK-16, } \\
\text { GMA-17, GOK-18, GOK-19, GAP-20, GMF-21, GMI-22, GMS-24, } \\
\text { GNK-25, GNU-26, GNF-27, GKT-29, GKK-30, GMR-31, GAD-32, } \\
\text { GNP-33, GBC-34, GJG-35, GNP-36. }\end{array}$ \\
\hline \multirow{4}{*}{16} & \multirow{4}{*}{ Bract tip: colour } & \multirow{4}{*}{ VS } & White & 1 & 00 & - \\
\hline & & & Rose & 3 & 33 & $\begin{array}{l}\text { GKM-1, GKM-2, GKB-3, GKB-4, GKJ-5, GMV-6, GBS-8, GBH-9, } \\
\text { GGR-10, GAB-11, GAB-12, GAB-13, GAB-14,GMW-15, GMK-16, } \\
\text { GMA-17, GOK-18, GOK-19, GAP-20, GMF-21,GMI-22, GMS-24, } \\
\text { GNK-25, GNU-26, GNF-27, GKT-29, GKK-30, GMR-31, GAD-32, } \\
\text { GNP-33, GBC-34, GJG-35, GNP-36. }\end{array}$ \\
\hline & & & Purple & 5 & 00 & - \\
\hline & & & Green & 7 & 00 & - \\
\hline \multirow{3}{*}{17} & \multirow{3}{*}{ Rhizome: Habit } & \multirow{3}{*}{ VG } & Compact & 3 & 12 & $\begin{array}{l}\text { GKM-1, GKB-3, GMV-6, GGR-10, GAB-12, GMA-17, GAP-20, GMS- } \\
\text { 24, GNK-25, GNU-26, GNF-27, GAD-32, }\end{array}$ \\
\hline & & & \begin{tabular}{|l} 
Intermediate \\
\end{tabular} & 5 & 06 & GKJ-5, GMW-15, GOK-18, GMI-22, GJG-35, GNP-36. \\
\hline & & & Loose & 7 & 15 & $\begin{array}{l}\text { GKM-2, GKB-4, GBS-8, GBH-9, GAB-11, GAB-13, GAB-14, GMK-16, } \\
\text { GOK-19, GMF-21, GKT-29, GKK-30, GMR-31, GNP-33, GBC-34. }\end{array}$ \\
\hline
\end{tabular}

\begin{tabular}{|c|c|c|c|c|c|c|}
\hline $\begin{array}{l}\text { SI } \\
\text { no }\end{array}$ & Characteristic & $\begin{array}{c}\text { Type of } \\
\text { Assessment }\end{array}$ & States & Score & $\begin{array}{c}\text { No. of } \\
\text { genotypes }\end{array}$ & Reference genotypes \\
\hline 18 & MS & Straight & 3 & 6 & GKB-4, GMV-6, GAB-13, GAB-14, GMF-21, GMI-22, \\
\cline { 3 - 7 } & Rhizome: shape & Curved & 5 & 27 & $\begin{array}{c}\text { GKM-1, GKM-2, GKB-3, GKJ-5, GBS-8, GBH-9, GGR-10, GAB-11, } \\
\text { GAB-12, GMW-15, GMK-16, GMA-17, GOK-18, GOK-19, GAP-20, } \\
\text { GMS-24, GNK-25, GNU-26, GNF-27, GKT-29, GKK-30, GMR-31, } \\
\text { GAD-32, GNP-33. GBC-34, GJG-35, GNP-36. }\end{array}$ \\
\hline 19 & $\begin{array}{c}\text { Rhizome: Primary } \\
\text { rhizome length } \\
(\mathrm{cm})\end{array}$ & MS & $\begin{array}{c}\text { Medium }(5- \\
10)\end{array}$ & 5 & 29 & $\begin{array}{c}\text { GKM-1, GKB-4, GMV-6, GBS-8, GBH-9, GGR-10, GAB-11, GAB-12, } \\
\text { GAB-13, GAB-14,GMW-15, GMK-16, GMA-17, GOK-18, GOK-19, } \\
\text { GAP-20, GMF-21, GMS-24, GNK-25, GNU-26, GNF-27, GKT-29, }\end{array}$ \\
\cline { 3 - 7 }
\end{tabular}




\begin{tabular}{|c|c|c|c|c|c|c|}
\hline & & & & & & GKK-30, GMR-31, GAD-32, GNP-33. GBC-34, GJG-35, GNP-36. \\
\hline & & & Long $(>10)$ & 7 & 04 & GKM-2, GKB-3, GKJ-5, GMI-22, \\
\hline & & & One & 1 & 00 & - \\
\hline & & & Two- three & 3 & 00 & - \\
\hline 20 & $\begin{array}{l}\text { Rhizome: Number } \\
\text { of mother rhizomes }\end{array}$ & MG & $\begin{array}{l}\text { More than } \\
\text { Three }\end{array}$ & 5 & 33 & $\begin{array}{l}\text { GKM-1, GKM-2, GKB-3, GKB-4, GKJ-5, GMV-6, GBS-8, GBH-9, } \\
\text { GGR-10, GAB-11, GAB-12, GAB-13, GAB-14,GMW-15, GMK-16, } \\
\text { GMA-17, GOK-18, GOK-19, GAP-20, GMF-21,GMI-22, GMS-24, } \\
\text { GNK-25, GNU-26, GNF-27, GKT-29, GKK-30, GMR-31, GAD-32, } \\
\text { GNP-33, GBC-34, GJG-35, GNP-36. }\end{array}$ \\
\hline 21 & Rhizome: Internode & MS & Close $(<1)$ & 3 & 18 & $\begin{array}{l}\text { GKB-3, GKB-4, GBS-8, GBH-9, GAB-11, GAB-12, GAB-13, GAB-14, } \\
\text { GMW-15, GMK-16, GMA-17, GOK-18, GOK-19, GMF-21, GKK-30, } \\
\text { GMR-31, GJG-35, GNP-36. }\end{array}$ \\
\hline & & & Distant $(>1)$ & 5 & 15 & $\begin{array}{l}\text { GKM-1, GKM-2, GKJ-5, GMV-6, GGR-10, GAP-20, GMI-22, GMS-24, } \\
\text { GNK-25, GNU-26, GNF-27, GKT-29, GAD-32, GNP-33. GBC-34, }\end{array}$ \\
\hline & & & Absent & 1 & 10 & $\begin{array}{l}\text { GKB-4, GBS-8, GBH-9, GMW-15, GAP-20, GMF-21, GNK-25, GKT- } \\
\text { 29, GNP-33. GBC-34 }\end{array}$ \\
\hline 22 & $\begin{array}{l}\text { Rhizome: status of } \\
\text { tertiary rhizome }\end{array}$ & VG & present & 9 & 23 & $\begin{array}{c}\text { GKM-1, GKM-2, GKB-3, GKJ-5, GMV-6, GGR-10, GAB-11, GAB-12, } \\
\text { GAB-13, GAB-14, GMK-16, GMA-17, GOK-18, GOK-19, GMI-22, } \\
\text { GMS-24, GNU-26, GNF-27, GKK-30, GMR-31, GAD-32, GJG-35, } \\
\text { GNP-36. }\end{array}$ \\
\hline $\begin{array}{l}\text { SI } \\
\text { no }\end{array}$ & Chara & $\begin{array}{c}\text { Type of } \\
\text { Assessment }\end{array}$ & States & Score & $\begin{array}{c}\text { No. of } \\
\text { genotypes }\end{array}$ & Reference genotypes \\
\hline & & & Light blue & 1 & 12 & $\begin{array}{c}\text { GKM-2, GKB-3, GGR-10, GAB-11, GAB-13, GAB-14, GMA-17, GMI- } \\
\text { 22, GNP-33. GBC-34, GJG-35, GNP-36. }\end{array}$ \\
\hline & & & Greenish blue & 3 & 01 & GKJ-5. \\
\hline 23 & Rhizome: Inner & VS & $\begin{array}{l}\text { Moderate } \\
\text { blue }\end{array}$ & 5 & 03 & GKM-1, GAB-12, GOK-19. \\
\hline & & & Strong blue & 7 & 16 & $\begin{array}{l}\text { GKB-4, GBS-8, GBH-9, GMW-15, GMK-16, GOK-18, GAP-20, GMF- } \\
\text { 21, GMS-24, GNK-25, GNU-26, GNF-27, GKT-29, GKK-30, GMR-31, } \\
\text { GAD-32. }\end{array}$ \\
\hline & & & Pale green & 9 & 01 & GMV-6. \\
\hline & & & Short $(<210)$ & 3 & 01 & 3-13, GAB-14. \\
\hline & & & $\begin{array}{l}\text { Medium } \\
(211-240)\end{array}$ & 5 & 06 & GKB-4, GBH-9, GMA-17, GMS-24, GNK-25, GKT-29. \\
\hline 24 & $\begin{array}{c}\text { Duration (Number } \\
\text { of Days) }\end{array}$ & VG & Long $(>241)$ & 7 & 26 & $\begin{array}{l}\text { GKM-1, GKM-2, GKB-3, GKJ-5, GMV-6, GBS-8, GGR-10, GAB-11, } \\
\text { GAB-12, GMW-15, GMK-16, GOK-18, GOK-19, GAP-20, GMF-21, } \\
\text { GMI-22, GNU-26, GNF-27, GKK-30, GMR-31, GAD-32, GNP-33. } \\
\text { GBC-34, GJG-35, GNP-36. }\end{array}$ \\
\hline & & & Low $(<15)$ & 3 & 05 & GKB-4, GBH-9, GMK-16, GMA-17, GKT-29. \\
\hline 25 & Dry recovery $(\%)$ & MG & $\begin{array}{c}\text { Intermediate } \\
(15-20)\end{array}$ & 5 & 18 & $\begin{array}{l}\text { GBS-8, GGR-10, GAB-13, GMW-15, GOK-18, GOK-19, GAP-20, } \\
\text { GMF-21, GMI-22, GNK-25, GNU-26, GNF-27, GKK-30, GMR-31, } \\
\text { GNP-33. GBC-34, GJG-35, GNP-36. }\end{array}$ \\
\hline & & & High $(>20)$ & 7 & 10 & $\begin{array}{l}\text { GKM-1, GKM-2, GKB-3, GKJ-5, GMV-6, GAB-11, GAB-12, GAB-14, } \\
\text { GMS-24, GAD-32. }\end{array}$ \\
\hline
\end{tabular}

Note:

MG: Measurement by a single observation of a group of plants or parts of plants

MS: Measurement of a number of individual plants or parts of plants

VG: Visual assessment by a single observation of a group of plants or parts of plants

VS: Visual assessment by observations of individual plants or parts of plants

Table 3A: Morphological characterization of Black turmeric genotypes using scoring for vegetative characters

\begin{tabular}{|c|c|c|c|c|c|c|c|c|c|c|c|c|c|c|}
\hline Characters & $\begin{array}{l}\text { Plant: } \\
\text { Pseudo } \\
\text { stem } \\
\text { habit }\end{array}$ & $\begin{array}{c}\text { Plant } \\
\text { height } \\
(\mathrm{cm})\end{array}$ & $\begin{array}{l}\text { Plant: } \\
\text { No. of } \\
\text { shoots }\end{array}$ & $\begin{array}{c}\text { Plant: } \\
\text { No. of } \\
\text { leaves } \\
\text { on } \\
\text { main } \\
\text { shoot } \\
\end{array}$ & $\begin{array}{l}\text { Plant: Leaf } \\
\text { disposition }\end{array}$ & $\begin{array}{l}\text { Leaf: } \\
\text { Petiole } \\
\text { length } \\
(\mathrm{cm})\end{array}$ & $\begin{array}{l}\text { Leaf: } \\
\text { Lamina } \\
\text { length } \\
(\mathrm{cm})\end{array}$ & $\begin{array}{l}\text { Leaf: } \\
\text { Lamina } \\
\text { width } \\
(\mathrm{cm})\end{array}$ & $\begin{array}{c}\text { Leaf: } \\
\text { colour } \\
\text { on } \\
\text { dorsal } \\
\text { side }\end{array}$ & $\begin{array}{c}\text { Leaf: } \\
\text { colour } \\
\text { on } \\
\text { ventral } \\
\text { side }\end{array}$ & $\begin{array}{c}\text { Leaf: } \\
\text { Margin }\end{array}$ & $\begin{array}{c}\text { Leaf: } \\
\text { Ventaion } \\
\text { pattern }\end{array}$ & $\begin{array}{l}\text { Pseudostem: } \\
\text { Anthocyanin } \\
\text { colouration }\end{array}$ & $\begin{array}{c}\text { Leaf: } \\
\text { midrib } \\
\text { colour }\end{array}$ \\
\hline Genotypes & 1 & 2 & 3 & 4 & 5 & 6 & 7 & 8 & 9 & 10 & 11 & 12 & 13 & 14 \\
\hline GKM-1 & 1 & 3 & 5 & 5 & 3 & 5 & 5 & 5 & 5 & 5 & 3 & 5 & 1 & 1 \\
\hline GKM-2 & 1 & 7 & 5 & 5 & 3 & 7 & 7 & 7 & 3 & 5 & 3 & 5 & 1 & 3 \\
\hline GKB-3 & 1 & 7 & 5 & 5 & 3 & 7 & 7 & 7 & 3 & 7 & 3 & 5 & 9 & 3 \\
\hline GKB-4 & 9 & 3 & 3 & 3 & 5 & 3 & 3 & 3 & 3 & 5 & 3 & 3 & 9 & 1 \\
\hline GKJ-5 & 1 & 7 & 5 & 5 & 3 & 7 & 7 & 7 & 3 & 7 & 3 & 5 & 1 & 1 \\
\hline GMV-6 & 9 & 3 & 5 & 5 & 5 & 5 & 5 & 5 & 3 & 5 & 3 & 3 & 1 & 3 \\
\hline GBS-8 & 1 & 3 & 5 & 5 & 3 & 3 & 3 & 3 & 5 & 7 & 3 & 3 & 9 & 1 \\
\hline GBH-9 & 9 & 3 & 5 & 5 & 3 & 3 & 3 & 3 & 3 & 5 & 3 & 3 & 9 & 3 \\
\hline GGR-10 & 9 & 3 & 5 & 5 & 3 & 7 & 7 & 7 & 3 & 5 & 3 & 3 & 1 & 3 \\
\hline GAB-11 & 9 & 5 & 5 & 3 & 5 & 5 & 5 & 5 & 5 & 5 & 3 & 3 & 1 & 3 \\
\hline GAB-12 & 9 & 5 & 5 & 5 & 3 & 5 & 5 & 5 & 3 & 5 & 3 & 3 & 9 & 3 \\
\hline GAB-13 & 1 & 3 & 5 & 5 & 5 & 5 & 5 & 5 & 5 & 7 & 3 & 3 & 9 & 3 \\
\hline
\end{tabular}




\begin{tabular}{|c|c|c|c|c|c|c|c|c|c|c|c|c|c|c|}
\hline GAK-14 & 1 & 3 & 5 & 5 & 5 & 7 & 5 & 7 & 5 & 5 & 3 & 3 & 9 & 3 \\
\hline GMW-15 & 1 & 3 & 5 & 5 & 5 & 3 & 3 & 5 & 5 & 7 & 3 & 3 & 1 & 1 \\
\hline GMK16 & 9 & 3 & 5 & 5 & 5 & 3 & 3 & 3 & 3 & 5 & 3 & 3 & 9 & 3 \\
\hline GMA-17 & 9 & 3 & 5 & 5 & 5 & 7 & 5 & 7 & 3 & 5 & 3 & 3 & 1 & 3 \\
\hline GOK-18 & 9 & 3 & 5 & 3 & 5 & 5 & 5 & 5 & 3 & 5 & 3 & 3 & 1 & 3 \\
\hline GOK-19 & 9 & 5 & 5 & 5 & 5 & 7 & 7 & 7 & 3 & 5 & 3 & 3 & 1 & 3 \\
\hline GAP-20 & 9 & 5 & 5 & 5 & 5 & 3 & 3 & 3 & 3 & 5 & 3 & 3 & 1 & 3 \\
\hline GMF-21 & 9 & 3 & 5 & 5 & 5 & 3 & 3 & 5 & 3 & 5 & 3 & 5 & 1 & 3 \\
\hline GMI-22 & 1 & 7 & 5 & 5 & 3 & 7 & 7 & 7 & 3 & 5 & 3 & 5 & 9 & 5 \\
\hline GMS-24 & 1 & 3 & 5 & 5 & 5 & 7 & 7 & 7 & 3 & 7 & 3 & 3 & 1 & 1 \\
\hline GNK-25 & 9 & 3 & 5 & 5 & 7 & 5 & 5 & 5 & 5 & 5 & 3 & 3 & 1 & 1 \\
\hline GNU-26 & 9 & 3 & 5 & 5 & 7 & 3 & 3 & 3 & 5 & 7 & 3 & 3 & 1 & 3 \\
\hline GNF-27 & 9 & 3 & 5 & 5 & 5 & 7 & 7 & 7 & 5 & 5 & 3 & 3 & 1 & 1 \\
\hline GKT-29 & 9 & 3 & 5 & 5 & 5 & 5 & 5 & 5 & 5 & 5 & 3 & 3 & 1 & 1 \\
\hline GKK-30 & 9 & 3 & 5 & 5 & 5 & 3 & 3 & 3 & 5 & 5 & 3 & 3 & 1 & 5 \\
\hline GMR-31 & 1 & 7 & 5 & 5 & 5 & 7 & 7 & 7 & 5 & 5 & 3 & 3 & 9 & 5 \\
\hline GAD-32 & 9 & 3 & 5 & 5 & 7 & 5 & 5 & 5 & 5 & 5 & 3 & 3 & 1 & 1 \\
\hline GNP-33 & 1 & 3 & 5 & 5 & 5 & 3 & 3 & 3 & 5 & 5 & 3 & 3 & 1 & 3 \\
\hline GBC-34 & 9 & 3 & 5 & 5 & 5 & 3 & 3 & 3 & 5 & 5 & 3 & 3 & 1 & 3 \\
\hline GJG-35 & 9 & 3 & 5 & 5 & 7 & 5 & 5 & 5 & 5 & 7 & 3 & 3 & 1 & 3 \\
\hline GNP-36 & 9 & 3 & 5 & 5 & 5 & 5 & 5 & 5 & 5 & 5 & 3 & 3 & 1 & 1 \\
\hline
\end{tabular}

Table 3B: Morphological characterization of Black turmeric genotypes using scoring for flower and Rhizome characters

\begin{tabular}{|c|c|c|c|c|c|c|c|c|c|c|c|}
\hline Characters & $\begin{array}{l}\text { Coma } \\
\text { bract: } \\
\text { colour }\end{array}$ & $\begin{array}{l}\text { Bract } \\
\text { tip: } \\
\text { colour }\end{array}$ & $\begin{array}{c}\text { Rhizome: } \\
\text { Habit }\end{array}$ & $\begin{array}{l}\text { Rhizome: } \\
\text { shape }\end{array}$ & $\begin{array}{c}\text { Rhizome: } \\
\text { Primary } \\
\text { rhizome } \\
\text { length }(\mathrm{cm}) \\
\end{array}$ & $\begin{array}{c}\text { Rhizome: } \\
\text { Number of } \\
\text { mother } \\
\text { rhizomes } \\
\end{array}$ & $\begin{array}{c}\text { Rhizome: } \\
\text { Internode } \\
\text { pattern }(\mathrm{cm})\end{array}$ & $\begin{array}{l}\text { Rhizome: } \\
\text { status of } \\
\text { tertiary } \\
\text { rhizome } \\
\end{array}$ & $\begin{array}{l}\text { Rhizome: } \\
\text { Inner core } \\
\text { colour }\end{array}$ & $\begin{array}{c}\text { Duration } \\
\text { (Number } \\
\text { of Days) }\end{array}$ & $\begin{array}{c}\text { Dry } \\
\text { recovery } \\
(\%)\end{array}$ \\
\hline \begin{tabular}{|l|} 
Genotypes \\
\end{tabular} & 15 & 16 & 17 & 18 & 19 & 20 & 21 & 22 & 23 & 24 & 25 \\
\hline GKM-1 & 9 & 3 & 3 & 5 & 5 & 5 & 5 & 9 & 5 & 7 & 7 \\
\hline GKM-2 & 9 & 3 & 7 & 5 & 7 & 5 & 5 & 9 & 1 & 7 & 7 \\
\hline GKB-3 & 9 & 3 & 3 & 5 & 7 & 5 & 3 & 9 & 1 & 7 & 7 \\
\hline GKB-4 & 9 & 3 & 7 & 3 & 5 & 5 & 3 & 1 & 7 & 5 & 3 \\
\hline GKJ-5 & 9 & 3 & 5 & 5 & 7 & 5 & 5 & 9 & 3 & 7 & 7 \\
\hline GMV-6 & 9 & 3 & 3 & 3 & 5 & 5 & 5 & 9 & 9 & 7 & 7 \\
\hline GBS-8 & 9 & 3 & 7 & 5 & 5 & 5 & 3 & 1 & 7 & 7 & 5 \\
\hline GBH-9 & 9 & 3 & 7 & 5 & 5 & 5 & 3 & 1 & 7 & 5 & 3 \\
\hline GGR-10 & 9 & 3 & 3 & 5 & 5 & 5 & 5 & 9 & 1 & 7 & 5 \\
\hline GAB-11 & 9 & 3 & 7 & 5 & 5 & 5 & 3 & 9 & 1 & 7 & 7 \\
\hline GAB-12 & 9 & 3 & 3 & 5 & 5 & 5 & 3 & 9 & 5 & 7 & 7 \\
\hline GAB-13 & 9 & 3 & 7 & 3 & 5 & 5 & 3 & 9 & 1 & 3 & 5 \\
\hline GAK-14 & 9 & 3 & 7 & 3 & 5 & 5 & 3 & 9 & 1 & 3 & 7 \\
\hline GMW-15 & 9 & 3 & 5 & 5 & 5 & 5 & 3 & 1 & 7 & 7 & 5 \\
\hline GMK16 & 9 & 3 & 7 & 5 & 5 & 5 & 3 & 9 & 7 & 7 & 3 \\
\hline GMA-17 & 9 & 3 & 3 & 5 & 5 & 5 & 3 & 9 & 1 & 5 & 3 \\
\hline GOK-18 & 9 & 3 & 5 & 5 & 5 & 5 & 3 & 9 & 7 & 7 & 5 \\
\hline GOK-19 & 9 & 3 & 7 & 5 & 5 & 5 & 3 & 9 & 5 & 7 & 5 \\
\hline GAP-20 & 9 & 3 & 3 & 5 & 5 & 5 & 5 & 1 & 7 & 7 & 5 \\
\hline GMF-21 & 9 & 3 & 7 & 3 & 5 & 5 & 3 & 1 & 7 & 7 & 5 \\
\hline GMI-22 & 9 & 3 & 5 & 3 & 7 & 5 & 5 & 9 & 1 & 7 & 5 \\
\hline GMS-24 & 9 & 3 & 3 & 5 & 5 & 5 & 5 & 9 & 7 & 5 & 7 \\
\hline GNK-25 & 9 & 3 & 3 & 5 & 5 & 5 & 5 & 1 & 7 & 5 & 5 \\
\hline GNU-26 & 9 & 3 & 3 & 5 & 5 & 5 & 5 & 9 & 7 & 7 & 5 \\
\hline GNF-27 & 9 & 3 & 3 & 5 & 5 & 5 & 5 & 9 & 7 & 7 & 5 \\
\hline GKT-29 & 9 & 3 & 7 & 5 & 5 & 5 & 5 & 1 & 7 & 5 & 3 \\
\hline GKK-30 & 9 & 3 & 7 & 5 & 5 & 5 & 3 & 9 & 7 & 7 & 5 \\
\hline GMR-31 & 9 & 3 & 7 & 5 & 5 & 5 & 3 & 9 & 7 & 7 & 5 \\
\hline GAD-32 & 9 & 3 & 3 & 5 & 5 & 5 & 5 & 9 & 7 & 7 & 7 \\
\hline GNP-33 & 9 & 3 & 7 & 5 & 5 & 5 & 5 & 1 & 1 & 7 & 5 \\
\hline GBC-34 & 9 & 3 & 7 & 5 & 5 & 5 & 5 & 1 & 1 & 7 & 5 \\
\hline GJG-35 & 9 & 3 & 5 & 5 & 5 & 5 & 3 & 9 & 1 & 7 & 5 \\
\hline GNP-36 & 9 & 3 & 5 & 5 & 5 & 5 & 3 & 9 & 1 & 7 & 5 \\
\hline
\end{tabular}

Table 4A: Colour of the Leaf midrib as per RHS colour chart

\begin{tabular}{|c|c|c|}
\hline \multicolumn{2}{|c|}{ RHS Colour group } & Genotypes \\
\hline \multirow{4}{*}{ Fan2 } & Violet blue group N92A & GKM-1, GKB-4, GKJ-5, GBS-8, GMW-15, GMS-24, GNK-25, GNF-27, GKT-29, GAD-32, GNP-36. \\
\cline { 2 - 3 } & Purple group N77A, & GKM-2, GMV-6, GBH-9, GGR-10, GAB-11, GAB-12, GJG-35, \\
\cline { 2 - 3 } & Purple group N79A, & GKB-3, GAB-14, GMK-16, GMA-17, GOK-18, GOK-19, GAP-20, GNU-26, \\
\cline { 2 - 3 } & Purple group N79B, & GAB-13, \\
\hline
\end{tabular}


Table 4B: Colour of the Pseudostem anthocyanin as per RHS colour chart

\begin{tabular}{|c|c|c|}
\hline \multicolumn{2}{|c|}{ RHS Colour group } & Genotypes \\
\hline \multirow{4}{*}{ Fan2 } & Red purple group 59A, & GKB-3, GBH-9, GAB-12, GAB-13, GAB-14, GMK-16, GMR-31, \\
\cline { 2 - 3 } & Red purple group 71A, & GBS-8, \\
\cline { 2 - 3 } & Purple group N79B, & GMI-22, \\
\cline { 2 - 3 } & Purple group N77A, & GKB-4, \\
\hline \multirow{4}{*}{ Fan3 } & Yellow green group 146A, & GKM-1, GAD-32, GNP-33, GBC-34. \\
\cline { 2 - 3 } & \multirow{2}{*}{ Yellow green group 146B, } & GKJ-5, GGR-10, GAB-11, GMW-15, GMA-17, GOK-18, GAP-20, GNK-25, GNU-26, GNF-27, GKT- \\
& Yellow green group 146C, & 29, GKK-30, GJG-35. \\
\cline { 2 - 3 } & Green group 138A & GKM-2, GMV-6, GOK-19, GMF-21, GMS-24, \\
\cline { 2 - 3 } & & GNP-36. \\
\hline
\end{tabular}

Table 4C: Colour of the Primary rhizome inner core as per RHS colour chart

\begin{tabular}{|c|c|c|}
\hline \multicolumn{2}{|r|}{ RHS Colour group } & Genotypes \\
\hline \multirow{12}{*}{ Fan2 } & Blue group 101B, & GMK-16, GAP-20. \\
\hline & Blue group 102B & GAB-12 \\
\hline & Blue group $102 \mathrm{C}$, & GBH-9 \\
\hline & Blue group 102D, & GOK-19 \\
\hline & Blue group 104A, & GOK-18, GNF-27, GKK-30. \\
\hline & Blue group 104B, & GKB-4, GBS-8, GMW-15, GMF-21, GMS-24, GNK-25,GNU-26, GKT-29, GAD-32. \\
\hline & Blue group 104C, & GMR-31 \\
\hline & Blue group 106B, & GKB-3, GAB-11 \\
\hline & Blue group 106D, & GAB-14 \\
\hline & Blue group 107C, & GMA-17, GNP-36. \\
\hline & Blue group 107D, & GNP-33, GJG-35. \\
\hline & Blue group 108A. & GMI-22, GBC-34. \\
\hline \multirow{6}{*}{ Fan3 } & Blue green group 110D, & GAB-13 \\
\hline & Blue green group 112A, & GKM-2 \\
\hline & Blue green group 113C, & GGR-10 \\
\hline & Blue green group 115A, & GKM-1 \\
\hline & Blue green group $117 \mathrm{C}$, & GKJ-5, \\
\hline & Blue green group 124D & GMV-6, \\
\hline
\end{tabular}

\section{Conclusion}

Black turmeric genotypes were characterized as per DUS guidelines for qualitative and quantitative characters. Present study concluded that the available variation in the selected genotypes. Vigourness of the plant can be considered as a morphological character for selection of adoptable genotypes to local situations. GKM-2, GKB-3, GKJ-5, GMI-22, GOK19, GAP-20, GNF-27, GMR-31 has shown better vegetative growth viz., taller plants, longer petioles \& lamina length and leaf width, these results represents the adoptability of these genotypes to the local conditions. Pseudo stem anthocyanin colouration of GAB12, GMI22 and GNP31 is unique. Three genotypes exhibits greyed purple (dark purplish green) leafmidrib colour viz., GMI-22, GKK-30, GMR-31. These morphology characters can be considered as an identification marker for these genotype. All the genotypes had coloured coma bract and exhibited rose bract tip. These important charecteristics will be a unique morphology for identification of the species $C$. caesia Roxb. Variation in the inner core colour of black turmeric rhizomes and primary rhizomes have been emphasized. Greenish blue \& Pale green colour of the rhizome inner core can be use as a marker for identification of the GKJ5 \& GMV6 genotypes respectively. Genotypes GAB13 and GAB-14 are short duration type (less than 210 Days), this morphological characteristic can be a marker for respective genotypes. Apart from DUS characters, there were some noticeable variation in the selected genotypes with respect to collar girth of the pseudo stem, colour intensity of leaf midrib \& rhizome inner core colour and lodging of the plant at 220 DAS which can act as morphological markers for identification of genotypes. The variations analysed using DUS characters offers a bright scope for selection based on desirable morphological traits, which can be potential in utilization for trait specific selection.

\section{References}

1. Aarthi S, Suresh J, Prasath D. Morphological characterization of Indian turmeric (Curcuma longa L.) genotypes using DUS descriptor. J Plant. Crops. 2018; 46(3):173-179.

2. Anandaraj M, Prasath D, Kandiannan K, John Zachariah T, Srinivasan V, Jha AK, Singh BK et al. Genotype by environment interaction effects on yield and curcumin in turmeric (Curcuma longa L.). Indl. Crop. Prod. 2014; 53:358-364.

3. Arulmozhi DK, Sridhar N, Veeranjaneyulu A, Arora SK. Preliminary mechanistic studies on the smooth muscle relaxant effect of hydroalcoholic extract of Curcuma caesia Roxb. J. Herb. Pharma. 2006; 6:117-24.

4. Das S, Bordoloi PK, Phukan D, Singh S. Study of the anti-ulcerogenic activity of the ethanolic extracts of rhizome of Curcuma caesia against gastic ulcers in experimental animals. Asian. J Pharm. Clin. Res. 2012; 5:200-203.

5. Deb BC, Chakrobarty S. Evaluation of genetic variability and characterization of some elite turmeric genotypes in Terai Region in India. Int. J Curr. Micro. App. Sc. 2017; 6(5):2357-2366. 
6. Dev A. Characterization and evaluation of somaclones of ginger (Zingiber officinale Rosc.). M.Sc. (Hort.) thesis, Kerala Agricultural University, Thrissur, 2013, 112.

7. Gill R, Kalsi V, Singh A. Phytochemical investigation and evaluation of anthelmintic activity of Curcuma amada and Curcuma caesia - A comparative study. Inventi Impact: Ethnopharmacology Article ID-'Inventi: ep/412/11", Available from:

http://www.inventi.in/Article/ep/412/11.aspx, 2011.

8. Jayashreee, Kandiannan K, Prasath D, Sasikumar B, Senthil Kumar CM, Srinivasan V et al. Turmeric (Extension pamphlet). Indian Institute of Spices Research, Kozhikode, 2015, 12.

9. Karmakar I, Dolai N, Bala A, Haldar PK. Anxiolytic and CNS depressant activities of methanol extract of Curcuma caesia rhizome. Pharmaco. 2011; 2:738-747.

10. Mangla M, Shuaib M, Jain, Kashyap M. In-vitro evaluation of antioxidant activity of Curcuma caesia Roxb. Int. J Pharm. Sci. Res. 2010; 1:98-102.

11. Mishra R, Gupta AK, Lal RK, Jhang T, Banerjee N. Genetic variability, analysis of genetic parameters, character associations and contribution for agronomical traits in turmeric (Curcuma longa L.). Ind. Crop. Prod. 2015; 76:204-208.

12. Nair RR, Aarthi S. New approaches in turmeric (Curcuma longa L.) breeding. International Symposium on Biodiversity of medicinal Plants and Orchids; Emerging Trends and Challenges. 2018, 39-40.

13. Neeraja A, Swami DV, Prasanna Kumar B, Kiran Patro TSKK, Salomi Suneetha DR, Babu Rao B. Influence of different planting material and major nutrient application on yield attributes of turmeric (Curcuma longa L.). Int. J Curr. Micro. App. Sci. 2017; 6(7):422-428.

14. Neha B, Tiwari KL, Jadhav SK. A review on nonconventional Turmeric Curcuma caesia Roxb. Curr. Trend. Biotech. Pharm. 2014; 8(1):91-101.

15. Padmadevi K, Jeeva Jothi L, Ponnuswami V, Durgavathi, V, Rijwana Parveen I. Effect of different grades of rhizomes on growth and yield of turmeric (Curcuma longa L.)., Asian J Hort. 2012; 7(2):465-467.

16. Paliwal P, Pancholi SS, Patel RK. Pharmacognostic parameters for evalution of the rhizomes of Curcuma caesia. J Advan. Pharma. Tech. Res. 2011; 2:56-61.

17. PPV\& FRA. Guidelines for the Conduct of Test for Distinctiveness, Uniformity and Stability on turmeric (Curcuma longa L.). India. http://plantauthority.gov.in/ pdf/Turmeric.pdf, 2009,

18. Prasath D, Eapen SJ, Sasikumar B. Performance of turmeric (Curcuma longa) genotypes for yield and root knot nematode resistance. Ind. J Agri. Sci. 2016; 86(9):89-92.

19. Rajamma AG, Bai V, Nambisan B. Antioxidant and antibacterial activities of oleoresins isolated from nine Curcuma species. Phy. Pharma. 2012; 2:312-317.

20. Sharma GJ, Chirangini P, Kishor R. Gingers of Manipur: Diversity and potentials as bioresources. Gen. Res. Cr. Evol., 2011; 58(5):753-767.

21. Singh AP, Pandey VP, Rahman Sma, Pervez R. Genetic variability and character association in turmeric (Curcuma longa L.). Trend. Bio. Sci. 2012; 5(1):11-13.

22. Wikipedia., 2017, Curcuma caesia Roxb. https://en.wikipedia.org/w/index.php? title=Curcuma_caesia:01-04. 